\title{
Functional Recovery of Locomotive Behavior in the Adult Spinal Dog
}

\author{
Yasunobu Handa, Akira Naito, Shinju Watanabe, \\ Shigeru Komatsu and Yoshifusa Shimizu \\ Department of Anatomy, Shinshu University School of \\ Medicine, Matsumoto 390
}

\begin{abstract}
Handa, Y., Naito, A., Watanabe, S., Komatsu, S. and Shimizu, Y. Functional Recovery of Locomotive Behavior in the Adult Spinal Dog. Tohoku J. exp. Med., 1986, 148 (4), 373-384_Complete transection of the midthoracic spinal cord was performed on adult female dogs. Development of reflexes and locomotive movements in the hindlimbs after spinal transection was investigated over several months. In the earlier stages after the surgery, dogs showed flaccid paralysis of the hindlimbs. Within several weeks, muscle tone of the hindlimbs was gradually increased accompanied by development of flexion reflex with after-discharge in addition to monosynaptic reflexes. Alternating stepping movement also began to develop. Afterward, extensor thrust and crossed extension reflex were observed. Standing behavior of the hindlimbs was found after enough development of the extensor thrust and correct placement of the pads of the toes. Steady development of stepping and standing caused forward locomotion using fore- and hindlimbs. This walking behavior of the hindlimbs was never inhibited by additional spinal cord transection. These findings indicate that the isolated spinal cord caudal to the transected site is responsible for the locomotive behavior of the hindlimbs in adult spinal dogs. Spinal automatism and development of responses induced by afferent inflow from outside the cord may help to explain such functional recovery of the paralyzed hindlimbs.__ spinal cord; dog; paraplegia; locomotion
\end{abstract}

Complete damage of the spinal cord in humans results in irreversible paralysis of the body segments caudal to the lesion. In contrast, it is well known that neonatal non-primate mammals within some weeks after birth exhibit walking on all four limbs after complete spinal transection at the thoracic or lumbar segments (Shurrager and Dykman 1951; Stelzner et al. 1975; Weber and Stelzner 1977 ; Forssberg et al. 1980a, b; Eidelberg et al 1981; Smith et al. 1982). It was also reported earlier that adult carnivores, especially dogs, showed recovery of locomotive ability in the hindlimbs to stand and walk after complete transection of the spinal cord at lumbothoracic level (Eichhorst and Naunyn 1874 ; Freusberg 1874 ; Golz and Freusberg 1874). As to walking in adult spinal dogs, however, an opposite view was arrived at by some investigators (cf. Shurrager and Dykman 1951). It was mentioned that locomotion of adult spinal dogs was mainly Received December 6, 1985 ; accepted for publication March 19, 1986. 
achieved by the intact forelimbs and their hindlimbs played no important part in walking. Furthermore, Sherrington (1910) described that spinal dogs showed stepping and standing movements, but they never exhibited walking behavior. It was also described that spontaneous standing and walking were impossible in cats who were already adult at the time of transection (Eidelberg et al. 1980 ; Eidelberg 1981 ; Edgerton et al. 1983). Thus, it has been generally accepted that functional recovery of locomotive movements in spinal animals depends upon the age at the time of transection and almost no adult spinal animals become capable spontaneous walking with their hindlimbs.

In a brief communication described by Hart (1971), however, spontaneous walking for short distances has already been reported in two of eight adult spinal dogs. He emphasized that the dogs could stand without lowering the head as a counterweight indicating that the hindlimbs took part in the reflex standing. However, precise and systematic investigations about reflex walking in adult spinal dogs have not been reported yet.

We have investigated development of reflexes and locomotive movements in the hindlimbs of the adult dogs with spinal transection at T8-T10 over several months and found that most of them can stand and walk with their hindlimbs for considerable long distance. Therefore, the purpose of this study is to describe the spinal function restored after complete transverse transection of the spinal cord in adult dogs.

\section{Materials and Methods}

Nine mongrel dogs were selected and inspected for long-term observations following spinal transection. Only adult female dogs were chosen for easy evacuation of the bladder. The level of transection and duration of postoperative observation are shown in Table 1.

The dura and arachnoid were opened after exposure of the spinal cord by laminectomy

TABLE 1. Subjects, transection level and period of observation

\begin{tabular}{ccccccccc}
\hline \multirow{2}{*}{ No. } & \multirow{4}{*}{ Sex } & \multicolumn{3}{c}{ Transection level } & \multicolumn{3}{c}{ Locomotive movements } & Period of \\
\cline { 3 - 6 } & & 1st & 2nd & 3rd & Step & Stand & Walk & observation \\
\hline 1 & F & T10 & T 5 & & + & + & + & 24 (months) \\
2 & F & T 9 & & & + & + & - & 10 \\
3 & F & T 9 & & & + & + & + & 8 \\
4 & F & T 9 & T11 & T5 & + & + & + & 39 \\
5 & F & T 9 & & & + & + & + & 19 \\
6 & F & T 9 & & & + & + & - & 31 \\
7 & F & T 9 & & & + & + & + & $12^{*}$ \\
8 & F & T 9 & & & + & + & + & $11^{*}$ \\
9 & F & T 9 & & & + & + & + & 6 \\
\hline
\end{tabular}

* Further observations are in progress. 
under anesthesia with sodium pentobarbital $(25.9 \mathrm{mg} / \mathrm{kg}$ i.v.) in aseptic conditions. With great care not to injure the vessels on the dorsal surface of the pia, the pia was opened and stripped off the spinal cord. Then,the cord was transversely dissected until the entire inner surface of the pia was visible. In addition, spinal evoked potentials were checked to see if impulses could be conducted through the transected site.

After the surgery, all dogs were maintained in boxes filled with wood excelsior in an air-conditioned room. This kept them dry and clean, resulting in the best condition for preventing decubitus and infection. Penicillin was administered for two weeks. Three weeks after the surgery, all dogs were transferred to an observation room with a wide floor covered with excelsior. They could move freely throughout this room resulting in a good rehabilitation for the spinal function caudal to the transected site. No training programs for the restoration of spinal function were applied to the spinal dogs.

Muscle discharges of the gastrocnemius and quadriceps femoris were detected by a low noise differential amplifier developed by us with coiled stainless steel wire electrodes, modified from the Caldwell type electrode (Caldwell and Reswick 1975). These electrodes were percutaneously implanted into the muscles using the technique of Basmajian and Stecko (1962).

\section{Results}

Transection of the spinal cord at the level of the eighth or ninth thoracic vertebra resulted in cessation of movements in the body segment caudal to the lesion. However, great variability in muscle tone and reflexes in the hindlimbs was observed during 2 or 3 days after the surgery. Some dogs showed complete flaccidity and depression of segmental reflex immediately after recovery from anesthesia. In others, the knee and ankle jerks were slightly exaggerated concomitant with a moderate increase in muscle tone of the hindlimbs. None of them exhibited polysynaptic reflexes. At this stage, the hindlimbs tended to be dragged backward during locomotion by the forelimbs (Fig. 1a). Urinary retention occurred in all of the dogs, and, therefore, urine had to be eliminated once daily by squeezing the abdomen.

From 4 to 14 days after the spinal transection, the muscle tone was gradually increased day by day. Knee and ankle jerks were prominently exaggerated and radiated to the contralateral hindlimbs. Tail pinching sometimes caused a decrease in the hypertonicity of both the hindlimbs, while its release was followed by bilateral extension of the hindlimbs for 5 to $10 \mathrm{sec}$. Ipsilateral flexion reflex began to develop as noxious stimuli were applied to the foot pad. Accompanied by development of the flexion reflex, the effect of the stimuli was carried over after release of the stimuli, i.e., the after-discharge.

By 2 or 3 weeks after the surgery, the hindlimbs had reached their maximum muscle tone. Since muscle tone of the extensors was much more dominant than that of the flexors in the paralyzed limbs, they thrust their hindlimbs forward stiffly between or to one side of the forelimbs (Fig. 1b). At the same time automatic evacuation of the bladder began to develop. Alternating stepping movements of the hindlimbs were elicited when the dogs were hung up. At the beginning, amplitude and frequency of the movements were small and low, 


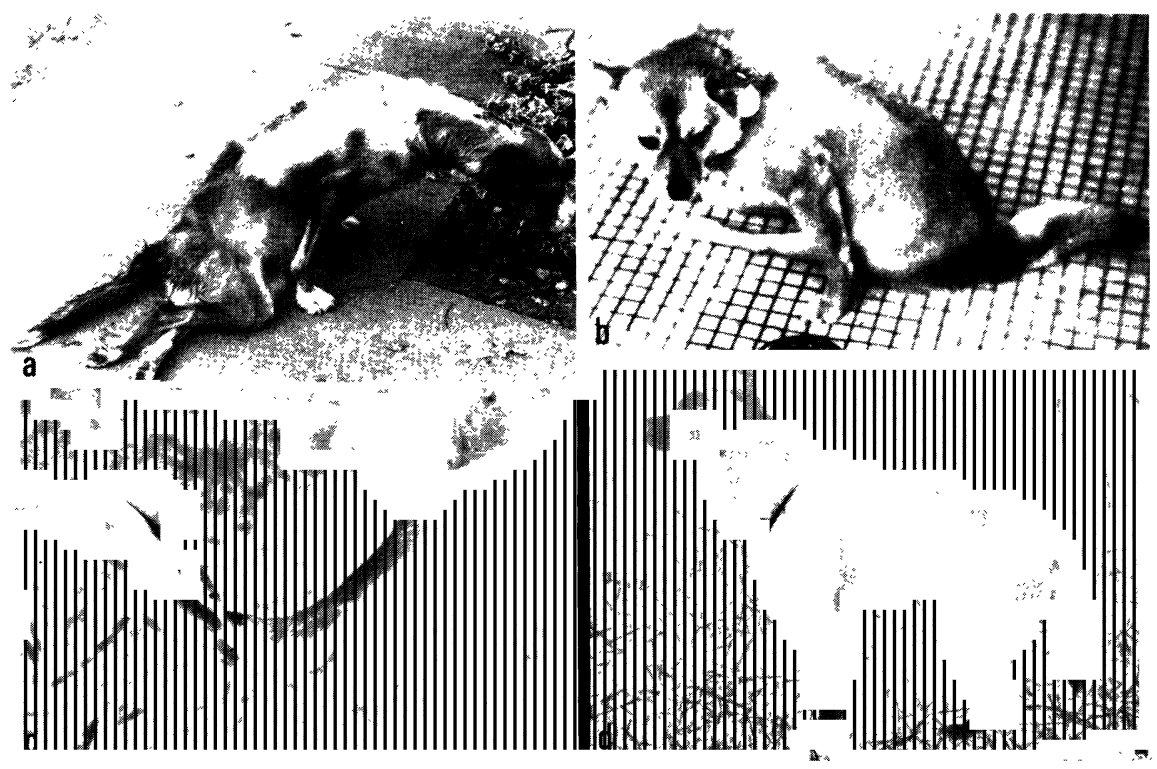

Fig. 1. Functional changes of the hindlimbs after spinal cord transection at T9. $a$, three days after spinal transection; $b$, forward extension of the both hindlimbs due to hypertonicity of the extensors ( 10 days post-operatively); c, extensor thrust reflex; d, standing with head up.

respectively. As days passed, the stepping became greater in amplitude and frequency.

By one or two months after the surgery, the excessive extensor hypertonicity was considerably decreased. Most of the reflexes developed well at this stage. The ipsilateral flexion reflex with longer period of after-discharge could easily be elicited by pinching the foot pad. Furthermore, sustained pinching caused extension of the contralateral hindlimb, i.e., the crossed extension reflex. In addition, extensor thrust reflex began to develop, induced by applying pressure to the foot pad to dorsiflex the toes (Fig. 1c). In contrast, plantarflexion of the toes resulted in a marked decrease in the extensor tone of the limb. Although the bilateral hindlimbs tended to flex into a more sitting posture, the paws never touched the ground because of the plantarflexion of the toes. Therefore, standing behavior of the hindquarters could not observed during this period.

After 3 months, further developments of the reflexes and movements were shown. Flexion reflex and crossed extension reflex were more pronounced and were accompanied by markedly prolonged after-discharge. Upward pressure against the pads of the bilateral hindlimbs caused a reflex of the full extension of the entire hindlimbs. The force of this extensor thrust seemed to be enough to support the body weight by their hindlimbs. In fact, most of the dogs began to stand up with their hindlimbs from a sitting position. In the earlier stages, they stood utilizing the wall of the cage for support. Furthermore, it was also found 
that they lowered their head for raising the hindquarters. This suggests that such standing behavior is maintained by musculatures rostral to the transection. As days passed, they could rise their hindquarters on open ground with their head up (Fig. 1d). A primary requirement for this standing was correct placement of the pads of the toes in the proper position.

The stepping movement also well developed, i.e., its amplitude and frequency increased markedly. However, the stepping was easily modified by various kinds of stimulation. Pressure to the abdominal wall caused marked increases in amplitude and frequency of the alternating stepping movement (Fig. 2A) while pinching the abdominal skin suppressed the movement ipsilaterally. Tail pinching induced bilateral suppression of the stepping movement. However, this was not universaly the case. Reinforcement of the movement was also induced by the same maneuver in some dogs. Insertion of a needle into the quadriceps femoris resulted in cessation of the stepping movement of the ipsilateral hindlimb (Fig. 2B). Simultaneously, needle insertion markedly decreased extensor tone of the ipsilateral hindlimb resulting in inhibition of extensor thrust and crossed extension reflex. This inhibition occurred most likely when the needle penetrated the fascia of the muscle, thus indicating that afferent impulses from the fascia and/ or muscle may play some part in the inhibition of the some reflexes and stepping movement.

On the average, by 4 months after the surgery, spinal walking was achieved after a steady development of stepping and standing activities. Fig. 3a shows electromyographic data of the bilateral gastrocnemius of a spinal dog during walking movements induced by the following maneuver. In supporting posture of the forepart of the body by her forelimbs, the bilateral hindlimbs were put on the palms of the hands and were alternately brought for- and backward by the hands. During backward movement of the unilateral hindlimb, the limb was fully extended and the force of the extensors was enough to support the body weight. This may correspond with a stance phase of the limb during gait. Muscle discharges of the gastrocnemius showed the maximum interference pattern (dotted lines). In a certain angle of ipsilateral hip extension during the backward movement, the ankle, knee and hip joints of that limb were suddenly flexed and the discharges of the gastrocnemius were almost completely suppressed. This may be the onset of a swing phase of the limb. Forward movement of the hindlimb during the swing phase caused a gradual increase in its extensor tone as known from the EMG of the gastrocnemius (continuous lines). When the sudden flexion of the contralateral hindlimb was induced by a certain degree of hip extension, the ipsilateral hindlimb showed full extension and could support the body weight.

Concomitant with improvement in balance during locomotion, most of the spinal dogs could walk using their hindlimbs on open ground. Fig. 3b shows cinematographic pictures illustrating spinal walking in Dog No.4. She could 
A

$r t$

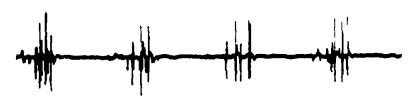

It

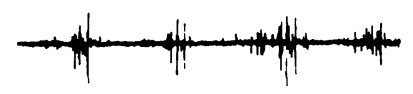

b

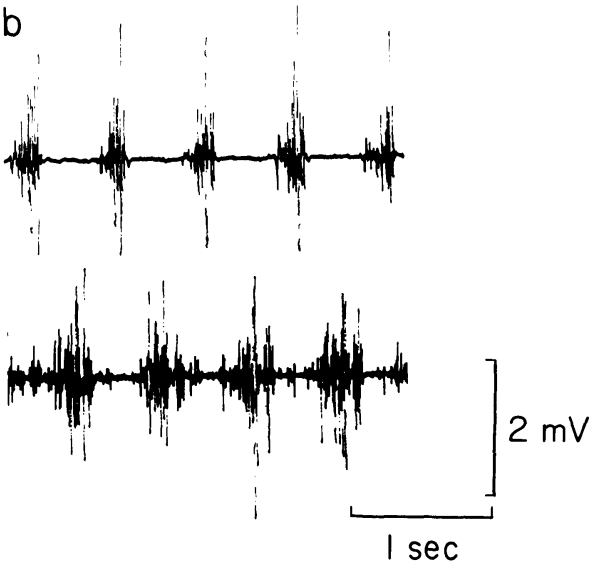

B

a<smiles>[3H]</smiles>

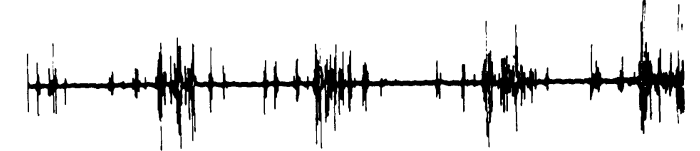

$1+$

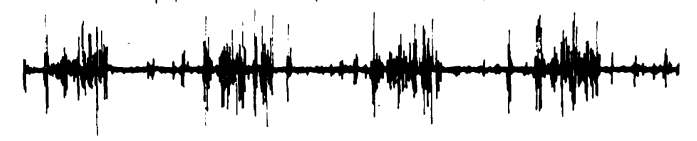

b

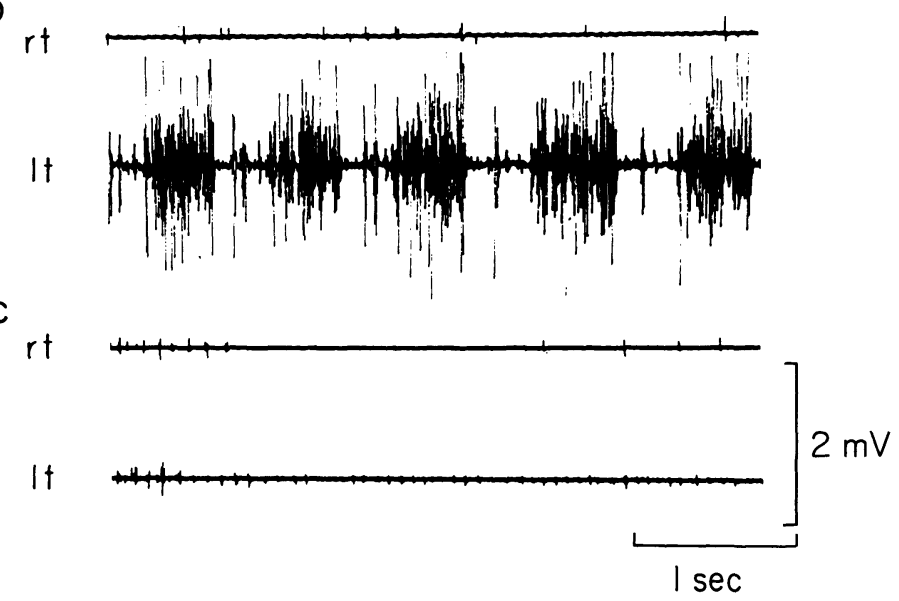

Fig. 2. Muscle discharges elicited in the bilateral quadriceps femoris during stepping movement. A shows reinforcement of the stepping (b) induced by pressure to the abdominal wall. a, control ; rt, discharges in the right side ; $1 t$, discharges in the left side. B illustrates effect of needle insertion into the quadriceps femoris. EMG was detected from the quadriceps femoris. a, before needle insertion; $b$, needle insertion to the right quadriceps femoris (rt). Discharges from the right quadriceps femoris were almost completely suppressed while amplitude and frequency of muscle discharges from the left quadriceps femoris (lt) increased markedly. c, complete suppression of muscle discharges induced by needle insertion to the bilateral quadriceps femoris. 

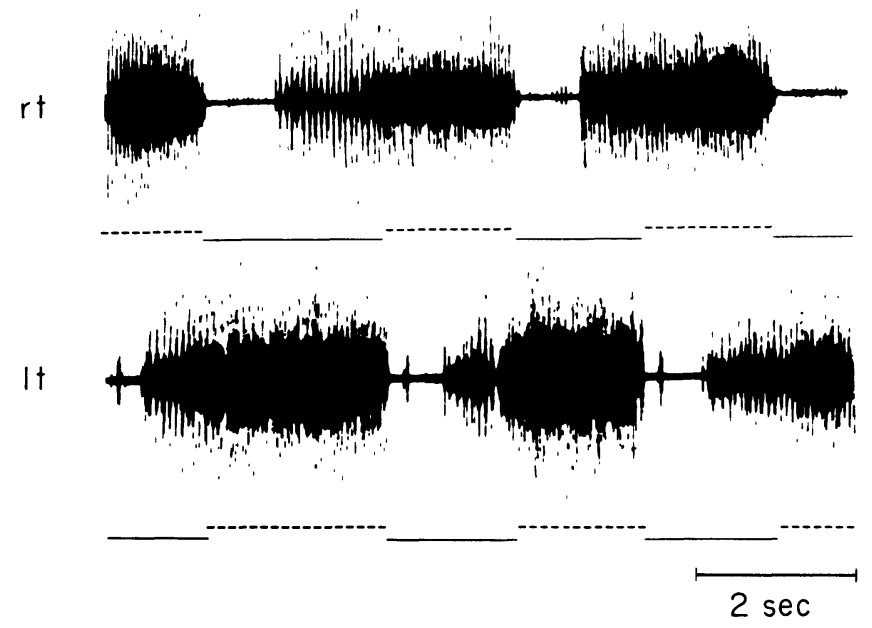

a

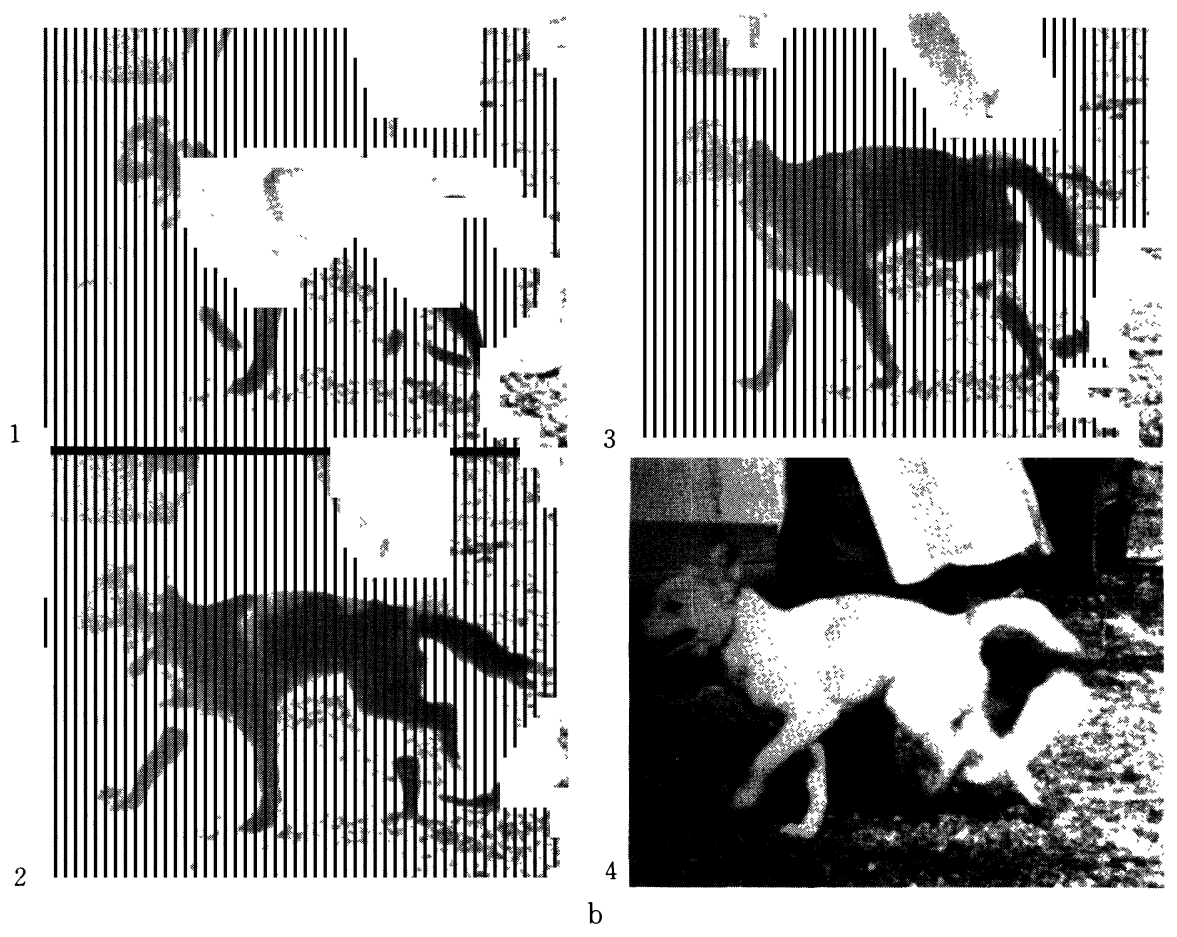

Fig. 3. Spinal walking in adult dogs with spinal cord transection at T9. a, EMG recordings from the gastrocnemius during walking behavior in the hind limbs. The hindlimbs were alternately brought forward (continuous lines) and backward (dotted lines). b, cinematographic pictures (1-4) showing spinal walking on open ground. 
walk more than $5 \mathrm{~m}$ with her head up. Coordination between the fore- and hindlimbs, however, seemed to be imperfect. In addition, the amplitude of the angular movement of the joints of the hindlimbs apparently increased during locomotion as compared with that of normal dogs. Therefore, they sometimes threw off balance in the case of rapid or long distance forward locomotion. This ability of locomotion by the hindlimbs of the spinal dogs reached a plateau 6 months after the surgery. The walking movement of the hindlimbs, however, was suppressed by noxious stimuli to the hindlimbs. Needle insertion into the quadriceps femoris still induced a marked decrease in extensor tone of the ipsilateral hindlimb which resulted in suppression of locomotive movements of that limb. Yet, the locomotion of the contralateral intact limb which was not inserted a needle was never inhibited to the extent that the dog could not stand and walk by taking advantage of the intact hindlimb. When a noxious stimulus was applied to muscles of both the hindlimbs, the hindlimbs were dragged passively during forward locomotion similar to those in dogs with spinal shock.

From the results mentioned above, the question remained open whether either of supraspinal control by incomplete transection or reinnervation through transected site existed. Two of the spinal dogs were retransected at T5 (No. 1) and T11 (No. 4) to solve this quesion. Despite complete transection of the cord, any spinal shock and depression of spinal function due to the retransection was not observed. The hindlimbs were as responsive as before the retransection, including crossed extension reflex, extensor thrust, correct placement of the feet and stepping movements. Furthermore, they could stand and walk with their hindlimbs immediately after recovery from the second surgery. Of these dogs, No. 4 was given a third transection at the level of T5 in order to reconfirm that no collateral pathways existed beyond the gap of the transected sites. The result of

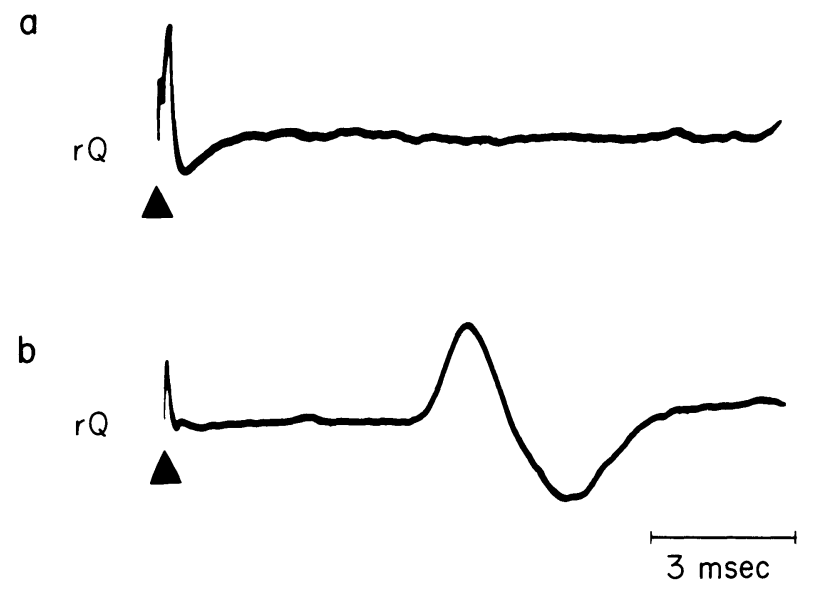

Fig. 4. EMG recordings from the right quadriceps femoris (rQ) evoked by stimulation (arrows) of the spinal cord rostral (a) and caudal (b) to the transected site. 
the third transection at $\mathrm{T} 5$ was the same as that of the second transection.

Electrophysiological data also supported that functional continuity of motor pathways did not occur from proximal to distal stumps of the transected spinal cord. The electromyography in Fig. 4. were recorded using the chronic spinal dog that could stand and walk. As shown in this figure, stimulation of the spinal cord rostral to the lesion never evoked discharges of the quadriceps femoris (a) and the subsequent contraction of the hindlimb muscles. In contrast, stimulation of the spinal cord caudal to the lesion evoked apparent muscle discharges (b) concomitant with strong contraction of the muscles.

Long-term observations over a period of 2 years made it clear that any reaction indicating the existence of supraspinal control and/or any sensory recovery were never observed in the dogs investigated. Locomotive behavior, once acquired, was maintained throughout the experiment, although any further development of the locomotion was not visible. However, one exception was observed in spinal Dog No.5. Extensor tone of her hindlimbs was markedly increased after she began spinal walking. Her walking and standing became worse as the muscle tone increased beyond the point of its maximum utility. Gradually, her hindlimbs exhibited forward extension due to dominant extensor tone of the hindlimbs. It became impossible for her to stand and walk with her hindlimbs by the times of 9 months after the surgery.

\section{Discussion}

Our results show that locomotive behavior, such as stepping, standing and walking, can be elicited in dogs with a completely transected spinal cord at the thoracic level. Electrophysiological investigations make the possibility remote that functional neural connections may exist across the gap between both stumps of the cord. Those investigations disprove the existence of any spinal nerves overlapping and communicating from the cord rostral to the lesion to the hindlimb muscles. In addition, the fact that the second and third transections have little effect on walking behavior once acquired further supports the view that the spinal cord caudal to the lesion is functionally isolated from the higher central nervous system. Similar results were also reported by Hart (1971).

Ranson and Hinsey (1930) described that the muscle tone of the hindlimbs was more marked in adult spinal dogs than in adult spinal cats. In adult spinal cats, spontaneous hindquarter standing was impossible, while pinching the tail resulted in short-lived standing due to an increase in extensor tone of the hindlimb (Ranson and Hinsey 1930; Eidelberg et al. 1980). In contrast, spontaneous standing is very common in spinal dogs as described by many investigators (Sherrington 1910; McCouch 1947; Shurrager and Dykman 1951). Our experiment showed that extensor thrust became well-developed before the spinal dogs were able to stand or walk. We also observed that proper placement of the plantar surface of the hindlimbs, as also observed by McCouch (1947), seemed to 
induce the extensor thrust reflex thus resulting in standing. This reflex is ipsilaterally inhibited by application of noxious stimuli to the quadriceps femoris and/or its fascia. Bilateral inhibition of the reflexes by insertion caused falling of the hindquarters and never elicited spinal standing. Excessive hypertonicity of the extensors also caused the inhibition of locomotive behavior as observed in Dog No. 5. Thus, muscle tone of the extensors in the hindlimbs seems to be one of the important factors for standing and other locomotive movements of spinal animals.

Shurrager and Dykman (1951) mentioned that forward locomotion by a forelimb induced a first step of the contralateral hindlimb in spinal carnivores. Grillner and Rossignol (1978) and Andersson et al. (1978) pointed out that a certain degree of hip extension resulted in a swing of the ipsilateral hindlimb and, thus, initiated walking movement. Our results corresponded with their data. It seems unlikely, however, that the hip angle is the only factor responsible for the initiation of a step. For this reason, the swing movement of a sufficiently extended hindlimb is not triggered until the contralateral limb is in a position to support body weight (Grillner and Rossignol 1978). On the other hand, our results showed that one hindlimb exhibited stepping and walking behavior while the other limb remained inactive. This indicates that phasic afferent inflow from the contralateral hindlimb are not always necessary for the performance of unilateral stepping and walking movements. Furthermore, it is known that stepping movements persist in preparation with multiple transection of the dorsal roots and/or immobilized by curarization (Grillner and Zangger 1974, 1979). These results suggest that segmental reflex is not a main factor in spinal stepping which seems to be essential for spinal locomotion. It seems likely, as described by many investigators (Andersson et al. 1978; Grillner and Zangger 1979; Eidelberg et al. 1980 ; Eidelberg 1983), that segmental step generators within an isolated portion of spinal cord are basically responsible for the stepping. It is also likely that segmental reflex inputs alter the amplitude, frequency and timing of the stepping movements of the hindlimbs.

Several investigators have pointed out that functional restoration of the spinal cord isolated from the higher central nervous system depends upon the age of the animal at the time of transection (Shurrager and Dykman 1951; Stelzner et al. 1975; Weber and Stelzner 1977; Eidelberg 1981; Smith et al. 1982 ; Edgerton et al. 1983). Stelzner et al. (1975) and Weber and Stelzner (1977) examined the effects of spinal transection on the development of locomotive behavior in neonatal and weanling rats. They concluded that locomotive movements were not possible in weanling rats, while the locomotive maturation of the hindlimbs in the neonatally transected rats resembled the ontogeny of locomotion in the normal rats. Shurrager and Dykman (1951) also reported that neonatal carnivores with spinal transection could walk with their hindlimbs after receiving electrical training. All of the above-mentioned investigators described that the 
critical period for initiation of spinal walking came at about 2 weeks after birth. Possible explanations are, as described by Stelzner et al. (1975, 1979), that development of local circuitry in the transected cord before functional supraspinal connections have formed and facilitation produced by an increased number of dorsal root afferents after transection plays an important role for the behavioral recovery of neonatal spinal animals.

However, such plastic activity of the spinal cord is also shown in the adult central nervous system (cf. Stelzner et al. 1975; Goldberger and Murray 1978; Cotman 1983). Although kinematic development of spinal locomotion is much slower in adult spinal dogs than in neonatal spinal animals, the developmental process, including the sequence of emergence of reflexes, is almost the same in both spinal animals. In addition, comparison of kinematic analysis reveals no significant difference between spinal locomotion in adult and neonatal spinal animals (Naito et al. unpublished). Therefore, it seems likely that the plastic activity as described in the neonatal spinal animals is also one of the important factors for the development of locomotion in adult spinal dogs.

Consequently, the spinal locomotion of adult dogs may be fundamentally attributed to the central step generator which is thought to exist inherently in the spinal cord, and to development of reflex activities induced by tonic afferent inflow.

\section{Acknowledgments}

This work was partly supported by Grant-in-Aid for Scientific Research from the Ministry of Education, Science and Culture of Japan (No. 57570021) and Special Fund from the Science and Technology Agency of Japan for promoting multiministrial projects 19731975 on 'the studies of regeneration of the nervous tissue'. Thanks to Tomofusa Nakano, Michio Miyata and Kumiko Yokouchi for their excellent assistance.

\section{References}

1) Andersson, O., Grillner, S., Lindquist, M. \& Zomlefer, M. (1978) Peripheral control of the spinal pattern generators for locomotion in cat. Brain Res., 150, 625-630.

2) Basmajian, J.V. \& Stecko, B.A. (1962) A new bipolar indwelling electrode for electromyography. Appl. Physiol., 17, 849.

3) Caldwell, C.W. \& Reswick, J.B. (1975) A percutaneous wire electrode for chronic research use. IEEE Trans. biomed. Eng., 22, 429-432.

4) Cotman, C.W. (1983) Reactive synaptogenesis in the adult CNS. In: Nerve, Organ, and Tissue Regeneration: Research Perspectives, edited by F.J. Seil, Academic Press, New York, pp. 269-282.

5) Edgerton, V.R., Johnson, D.J., Smith, L.A., Murphy, K., Eldred, E. \& Smith, J.L. (1983) Effects of treadmill exercises on hind limb muscles of the spinal cat. In: Spinal Cord Reconstruction, edited by C.C. Kao, R.P. Bunge \& P.J. Reier, Raven Press, New York, pp. 435-443.

6) Eichhorst, H. \& Naunyn, B. (1874) Über die Regeneration und Veränderungen im Rückenmarke nach Streckenweiser totaler Zerstörung desselben. Naunyn-Schmiedeberg's Arch. exp. Path. Pharmak., 2, 225-253.

7) Eidelberg, E. (1981) Consequences of spinal cord lesions upon motor function, with 
special reference to locomotor activity. Prog. Neurobiol., 17, 185-202.

8) Eidelberg, E. (1983) Loss and recovery of locomotor function after spinal cord lesions in cats and monkeys. In: Nerve, Organ, and Tissue Regeneration: Research Perspectives, edited by F.J. Seil, Academic Press, New York, pp. 231-242.

9) Eidelberg, E., Story, J.L., Meyer, B.L. \& Nystel, J. (1980) Stepping by chronic spinal cats. Exp. Brain Res., 40, 241-246.

10) Forssberg, H., Grillner, S. \& Halbertsma, J. (1980a) The locomotion of the low spinal cat. I. Coordination within a hindlimb. Acta physiol. scand., 108, 269-281.

11) Forssberg, H., Grillner, S., Halbertsma, J. \& Rossignol, S. (1980b) The locomotion of the low spinal cat. II. Interlimb coordination. Acta physiol. scand., 108, 283-295.

12) Freusberg, A. (1874) Reflexbewegungen beim Hunde. Pflügers Arch. ges. Physiol., 9, 358-391.

13) Goldberger, M.E. \& Murray, M. (1978) Recovery of movement and axonal sprouting may obey some of the same laws. In: Neuronal Plasticity, edited by C.W. Cotman, Raven Press, New York, pp. 73-97.

14) Goltz, F. \& Freusberg, A. (1874) Über Functionen des Lendenmarkes des Hundes. Pflügers Arch. ges. Physiol., 8, 460-482.

15) Grillner, S. \& Rossignol, S. (1978) On the initiation of the swing phase of locomotion in chronic spinal cats. Brain Res., 146, 269-277.

16) Grillner, S. \& Zangger, P. (1974) Locomotor movements generated by the deafferented spinal cord. Acta physiol. scand., 91, 38-39A.

17) Grillner, S. \& Zangger, P. (1979) On the central generation of locomotion in the low spinal cat. Exp. Brain Res., 34, 241-261.

18) Hart, B.L. (1971) Facilitation by strychnine of reflex walking in spinal dogs. In: Physiology and Behavior, Vol 6, Pergamon Press, New York, pp. 627-628.

19) McCouch, G.P. (1947) Reflex development in the chronically spinal cat and dog. $J$. Neurophysiol., 10, 425-428.

20) Ranson, S.W. \& Hinsey, J.C. (1930) Reflexes in the hind limbs of cats after transection of the spinal cord at various levels. Amer. J. Physiol., 94, 471-495.

21) Sherrington, C.S. (1910) Flexion-reflex of the limb, crossed exension-reflex, and reflex stepping and standing. J. Physiol., 40, 28-121.

22) Shurrager, P.S. \& Dykman, R.A. (1951) Walking spinal carnivores. J. comp. Physiol. Psychol., 44, 252-262.

23) Smith, J.L., Smith, L.A., Zernicke, R.F. \& Hoy, M. (1982) Locomotion in exercised and nonexercised cats cordotomized at two or twelve weeks of age. Exp. Neurol., 76, $393-413$.

24) Stelzner, D.J., Ershler, W.B. \& Weber, E.D. (1975) Effects of spinal transection in neonatal and weanling rats: Survival of function. Exp. Neurol., 46, 156-177.

25) Stelzner, D.J., Weber, E.D. \& Prendergast, J. (1979) A comparison of the effect of mid-thoracic spinal hemisection in the neonatal or weanling rat on the distribution and density of dorsal root axons in the lumbosacral spinal cord of the adult. Brain Res., 172, 407-426.

26) Weber, E.D. \& Stelzner, D.J. (1977) Behavioral effects of spinal cord transection in the developing rat. Brain Res., 125, 241-255. 